\title{
THE LAW OF LARGE NUMBERS, EXAMPLES AND COUNTEREXAMPLES
}

\author{
V. DOBRIC
}

In this paper we construct some examples and counterexamples that give answer to some open problems arising in the law of large numbers for nonseparable and non-measurable random variables, a theory which has recently been developed by J. Hoffman-Jørgensen [5] and E. Giné and J. Zinn [3].

I would like to thank J. Hoffmann-Jørgensen who has greatly influenced on the present paper.

Throughout all of this paper, $(S, \mathscr{S}, \mu)$ denotes a probability space, $B$ a Banach space, $B^{*}$ its dual and $\operatorname{LLN}(\mu, B)$ the set of all functions $f: S \rightarrow B$ which satisfy the following version of the law of large numbers (see [5])

$$
\text { (*) } \quad \exists a \in B: \lim _{n \rightarrow \infty}\left\|a-1 / n \sum_{i=1}^{n} f\left(s_{i}\right)\right\|=0 \text { for } \mu^{\infty}-\text { a.s. }\left(s_{j}\right) \in S^{\infty} \text {, }
$$

where $\left(S^{\infty}, \mathscr{S}^{\infty}, \mu^{\infty}\right)$ is the countable product of $(S, \mathscr{S}, \mu)$ with itself.

From [5] we know that if $f \in \operatorname{LLN}(\mu, B)$, then $\int^{*}\|f\| d \mu<\infty, f$ is Gelfand integrable and the $a$ occurring in (*) equals the Gelfand integral of $f$. M. Telegrand [6] has shown that if $f \in \operatorname{LLN}(\mu, B)$, then the convergence in (*) takes place in probability, too, and if $\int^{*}\|f\| d \mu<\infty$ and we have convergence in probability in $(*)$, then we also have convergence a. $s$.

We shall use the notation of [5], where the author considers the following four function spaces which we shall frequently use

$$
\begin{aligned}
L_{*}^{1}(\mu, B) & =\{f: S \rightarrow B \mid f \text { is weakly } \mu \text {-integrable }\} \\
L_{G}^{1}(\mu, B) & =\{f: S \rightarrow B \mid f \text { is Gelfand-integrable }\} \\
L^{1}(\mu, B) & =\{f: S \rightarrow B \mid f \text { is Bochner-integrable }\} \\
L_{*}^{1}(\mu, B) & =\left\{f: S \rightarrow B \mid \int^{*}\|f(s)\| \mu(d s)<\infty\right\} .
\end{aligned}
$$

Received June 04, 1985. 


\section{Examples of Banach spaces that satisfy LLN.}

First we shall prove two very useful lemmas. We let $c$ denote the cardinal of the continuum, i.e. $c=$ card $R$.

Lemma 1.1. Let $(S, \mathscr{S}, \mu)$ be a probability space, where $S$ is a Polish space with card $S=c, \mathscr{S}$ is the Borel $\sigma$-algebra on $S$ and $\mu$ is a diffuse probability measure. Then there exists a class $\mathscr{F}=\left\{F_{t} \mid t \in T\right\}$ of subsets of $S$ such that card $T=c$ and

$$
\begin{gathered}
\operatorname{card} F_{t} \leqq \aleph_{0}, \quad t \in T, \\
F_{t^{\prime}} \cap \dot{\boldsymbol{i}}_{t^{\prime \prime}}=\varnothing, \quad t^{\prime} \neq t^{\prime \prime}, \quad t^{\prime}, t^{\prime \prime} \in T,
\end{gathered}
$$

$\forall B \in \mathscr{S}^{\infty}$ with $\mu^{\infty}(B)>0, \quad \exists t \in T$ such that $B \cap F_{t}^{\infty} \neq \varnothing$,

$$
\bigcup_{t \in T} F_{t}=S
$$

Proof. Let $S$ be a Polish space with $\operatorname{card} S=c$. Then card $\mathscr{S}=c$ and also card $\mathscr{S}^{\infty}=c$. Let $\mathscr{S}_{+}=\left\{B \in \mathscr{S}^{\infty} \mid \mu^{\infty}(B)>0\right\}$. Then card $\mathscr{S}_{+}^{\infty}=c$. The set $S$ and the family $\mathscr{S}_{+}^{\infty}$ may be enumerated by cardinals less than $c$ : $\mathscr{S}_{+}^{\infty}=\left\{B_{t} \mid t<c\right\}$ and $S=\left\{s_{t} \mid t<c\right\}$. The set $B_{0} \in \mathscr{S}_{+}^{\infty}$ has positive measure and therefore $B_{0} \neq \varnothing$. Let us take $\left(s_{i}^{0}\right) \in B_{0}$ and define the set $\underline{F}_{0} \subseteq S$ by

$$
F_{0}= \begin{cases}\left\{s_{1}^{0}, s_{2}^{0}, \ldots, \ldots\right\} & \text { if } s_{0} \in\left\{s_{1}^{0}, s_{2}^{0}, \ldots, \ldots\right\} \\ \left\{s_{0}, s_{1}^{0}, s_{2}^{0}, \ldots, \ldots\right\} & \text { otherwise }\end{cases}
$$

Then card $F_{0} \leqq \aleph_{0}, F_{0}^{\infty} \cap B_{0} \neq \varnothing$ and $s_{0} \in F_{0}$. The set $\left(F_{0}^{c}\right)^{\infty}$ has $\mu^{\infty}$ measure 1 and therefore it intersects every set of positive $\mu^{\infty}$ measure, in particular $\left(F_{0}^{c}\right)^{\infty} \cap B_{1} \neq \varnothing$. Let us take $\left(s_{i}^{1}\right) \in\left(F_{0}^{c}\right)^{\infty} \cap B_{1}$ and define the set $F_{1} \subseteq S$ by

$$
F_{1}= \begin{cases}\left\{s_{1}^{1}, s_{2}^{1}, \ldots, \ldots\right\} & \text { if } s_{1} \in\left\{s_{1}^{1}, s_{2}^{1}, \ldots, \ldots\right\} \cup F_{0} \\ \left\{s_{1}, s_{1}^{1}, s_{2}^{1}, \ldots, \ldots\right\} & \text { otherwise }\end{cases}
$$

Then card $F_{1} \leqq \aleph_{0}, F_{1}^{\infty} \cap B_{1} \neq \varnothing, F_{1} \cap F_{0}=\varnothing$ and $s_{1} \in F_{0} \cup F_{1}$. By transfinite induction we shall finish our proof. To this end, suppose that the family $\left\{F_{t} \mid t<\alpha\right\}, \alpha<c$, has been constructed such that it satisfies (1.1.1)-(1.1.4) with $\alpha$ instead of $c$. Let

$$
F^{\alpha}=\bigcup_{t<\alpha} F_{t}
$$


We claim that

$$
B_{\alpha} \cap\left(\left(F^{\alpha}\right)^{c}\right)^{\infty} \neq \varnothing
$$

To show this, it is enough to prove that

$$
\left(\mu^{\infty}\right)^{*}\left(B_{\alpha} \cap\left(\left(F^{\alpha}\right)^{c}\right)^{\infty}>0 .\right.
$$

Borel sets in a Polish space are either countable or have cardinality $c$, and since

$$
\operatorname{card} F^{\alpha}=\operatorname{card} \bigcup_{t<\alpha} F_{t} \leqq \operatorname{card} \alpha \cdot \aleph_{0}<c,
$$

we conclude, because $\mu$ is diffuse, that

$$
\mu_{*}\left(F^{\alpha}\right)=0
$$

By Lemma 2.1 in [5], it then follows that

$$
\left(\mu^{\infty}\right)^{*}\left(\left(\left(F^{\alpha}\right)^{c}\right)^{\infty}\right)=1
$$

which implies (1.1.5) since $\mu\left(B_{\alpha}\right)>0$. Let us take $\left(s_{i}^{\alpha}\right) \in B_{\alpha} \cap\left(\left(F^{\alpha}\right)^{c}\right)^{\infty}$ and define the set $F_{\alpha} \cong S$ by

$$
F_{\alpha}= \begin{cases}\left\{s_{1}^{\alpha}, s_{2}^{\alpha}, \ldots, \ldots\right\} & \text { if } s_{\alpha} \in\left\{s_{1}^{\alpha}, s_{2}^{\alpha}, \ldots, \ldots\right\} \cup F^{\alpha} \\ \left\{s_{\alpha}, s_{1}^{\alpha}, s_{2}^{\alpha}, \ldots, \ldots\right\} & \text { otherwise }\end{cases}
$$

Then card $F_{\alpha} \leqq \aleph_{0}, F_{\alpha} \cap F_{t}=\varnothing, \forall t<\alpha, s_{\alpha} \in \bigcup_{t \leqq \alpha} F_{t}$ and $F_{\alpha}^{\infty} \cap B_{\alpha} \neq \varnothing$. Hence by transfinite induction (1.1.1)-(1.1.4) holds

Lemma 1.2. Let $(S, \mathscr{S}, \mu)$ be a probability space, where $S$ is a Polish space with card $S=c, \mathscr{S}$ is the Borel $\sigma$-algebra and $\mu$ is a diffuse probability measure. Then for every $n \in N$ there exists a class $\mathscr{F}_{n}=\left\{F_{t} \mid t \in T\right\}$ of subsets of $S$ such that $\operatorname{card} T=c$ and

$$
\begin{gathered}
\operatorname{card} F_{t} \leqq n+1, \quad \forall t \in T, \\
F_{t^{\prime}} \cap F_{t^{\prime \prime}}=\phi, \quad t^{\prime} \neq t^{\prime \prime}, \quad t^{\prime}, t^{\prime \prime} \in T,
\end{gathered}
$$$$
\forall B \in \mathscr{S}^{n} \text { with } \mu^{n}(B)>0, \exists t \in T \text { such that } F_{t}^{n} \cap B \neq \varnothing,
$$

$$
\bigcup_{t \in T} F_{t}=S \text {. }
$$


Proof. Replace $\mathscr{S}^{\infty}$ with $\mathscr{S}^{n}$ in the proof of Lemma 1.1.

TheOREM 1.3. Let $(S, \mathscr{S}, \mu)$ be a probability space, $T$ a set and $f: S \times T \rightarrow \mathrm{R}$ a stochastic process such that $f(s)=f(s, \cdot) \in l_{2}(T)$, for every $s \in S$. If the function $\eta: S \times S \rightarrow \mathrm{R}$ defined by

$$
\eta(s, v)=\sum_{t \in T} f(s, t) f(v, t) \quad \text { for }(s, v) \in S \times S
$$

is $\mu \times \mu$ measurable and

$$
\int_{s}^{*} \sqrt{\eta(s, s)} \mu(d s)<\infty
$$

then $f \in \operatorname{LLN}\left(\mu, l_{2}(T)\right)$.

Proof. Directly from the definition of $\eta$ it follows that

$$
|\eta(s, v)| \leqq \sqrt{\eta(s, s)} \sqrt{\eta(v, v)}, \quad \forall(s, v) \in S \times S,
$$

hence Fubini's theorem and (1.3.2) shows that $\eta \in L_{1}(S \times S, \mu \times \mu)$. Since

$$
T(s)=\{t \mid f(s, t) \neq 0\}
$$

is countable and

$$
\int_{S} \sum_{t \in T(s)}|f(s, t) f(v, t)| \mu(d v) \leqq \sqrt{\eta(s, s)} \int^{*} \sqrt{\eta(v, v)} \mu(d v)<\infty
$$

we have

$$
\begin{aligned}
\int_{S \times S} \eta d(\mu \times \mu) & =\int_{S} \mu(d s) \int_{S} \sum_{t \in T(s)} f(s, t) f(t, v) \mu(d v) \\
& =\int_{S} \sum_{t \in T} f(s, t) m(t) \mu(d s),
\end{aligned}
$$

where $m(t)=\int f(v, t) \mu(d v)$ is the mean of $f(\cdot, t)$. Hence by subtracting from $f$ 
its mean we may assume that

$$
\int_{S \times S} \eta d(\mu \times \mu)=0 .
$$

We then want to show that

$$
\lim _{n \rightarrow \infty}\left\|\frac{1}{n} \sum_{i=1}^{n} f\left(s_{i}\right)\right\|_{2}=0 \text { for } \mu^{\infty}-\text { a.s. }\left(s_{i}\right) \in S^{\infty} \text {. }
$$

The limit in (1.3.4) can be written in the following way

$$
\begin{aligned}
\lim _{n \rightarrow \infty}\left\|\frac{1}{n} \sum_{i=1}^{n} f\left(s_{i}\right)\right\|_{2}^{2} & =\lim _{n \rightarrow \infty}\left(\frac{1}{n} \sum_{i=1}^{n} f\left(s_{i}, t\right)\right)^{2} \\
& =\lim _{n \rightarrow \infty} \sum_{t \in T} \frac{1}{n^{2}} \sum_{i=1}^{n} \sum_{j=1}^{n} f\left(s_{i}, t\right) f\left(s_{j}, t\right) \\
& =\lim _{n \rightarrow \infty} \frac{1}{n^{2}} \sum_{i=1}^{n} \sum_{j=1}^{n} \eta\left(s_{i}, s_{j}\right) \\
& =\lim _{n \rightarrow \infty} \frac{1}{n^{2}} \sum_{i=1}^{n} \eta\left(s_{i}, s_{i}\right)+\lim _{n \rightarrow \infty} \frac{2}{n^{2}} \sum_{1 \leqq i<j \leqq n} \eta\left(s_{i}, s_{j}\right) .
\end{aligned}
$$

The last term in (1.3.5) is obtained by symmetry of $\eta$. The law of large numbers in [2, p. 122] applied to $X_{i}=$ the outer $\mu^{\infty}$-envelope of $\sqrt{\eta\left(\xi_{i}, \xi_{i}\right)}$, and condition (1.3.2) imply that

$$
\lim _{n \rightarrow \infty} \frac{1}{n^{2}} \sum_{i=1}^{n} \eta\left(s_{i}, s_{i}\right)=0 \text { for } \mu^{\infty}-\text { a.s. }\left(s_{i}\right) \in S^{\infty} .
$$

It remains to prove that the last term in (1.3.5) tends to $0 \mu^{\infty}-$ a.s. Let us define

$$
U_{n}=\frac{2}{n(n-1)} \sum_{1 \leqq i<j \leqq n} \eta\left(s_{i}, s_{j}\right) \quad \text { and } \quad \mathscr{F}_{n}=\sigma\left\{U_{i} \mid i \geqq n\right\} \quad \text { for } n \geqq 2 .
$$

Then for $n \geqq 2$ we have

$$
\begin{aligned}
U_{n}=E\left\{U_{n} \mid \mathscr{F}_{n}\right\} & =\frac{2}{n(n-1)} \sum_{1 \leqq i<j \leqq n} E\left\{\eta\left(s_{i}, s_{j}\right) \mid \mathscr{F}_{n}\right\} \\
& =E\left\{\eta\left(s_{1}, s_{2}\right) \mid \mathscr{F}_{n}\right\} .
\end{aligned}
$$


If we put $\mathscr{T}_{-n}=\mathscr{F}_{n}$ and $S_{-n}=E\left\{\eta\left(s_{1}, s_{2}\right) \mid \mathscr{F}_{n}\right\}$ for $n \geqq 2$, then $\left\{S_{n}, \mathscr{T}_{n},-\infty<n \leqq-2\right\}$ is a martingale and $E\left|S_{-2}\right|<\infty$ because $\eta \in L^{1}(\mu \times \mu)$. If

$$
\mathscr{T}_{-\infty}=\bigcap_{n=2}^{\infty} \mathscr{F}_{n},
$$

then Theorem 11.1.1 in $[2$, p. 376] ensures that

$$
\begin{aligned}
& \lim _{n \rightarrow \infty} S_{n} \quad\left(\text { say } S_{-\infty}\right) \quad \text { exists } \mu \times \mu-\text { a.s., } \\
& S_{-\infty} \text { is } \mathscr{T}^{-\infty} \text { measurable, } \\
& E\left|S_{-\infty}\right|<\infty
\end{aligned}
$$

Since $\mathscr{T}_{-\infty}$ is $\sigma$-algebra of permutable events, the Hewitt-Savage zero-one law (see [2, Corollary 7.3.8]) ensures that $S_{-\infty}$ is a constant random variable which coincide with $E \eta$. But by (1.3.3) $E \eta=0$ and therefore

$$
\lim _{n \rightarrow \infty} U_{n}=0
$$

Consequently

$$
\lim _{n \rightarrow \infty} \frac{1}{n^{2}} \sum_{1 \leqq i<j \leqq n} \eta\left(s_{i}, s_{j}\right)=\lim _{n \rightarrow \infty} \frac{n-1}{n} U_{n}=0 \text { for } \mu^{\infty}-\text { a.s. }\left(s_{i}\right) \in S^{\infty} \text {. }
$$

Theorem 1.3 gives a sufficient condition for $f$ to belong to $\operatorname{LLN}\left(\mu, l_{2}(T)\right)$ and naturally it brings us to the question whether the condition of Theorem 1.3 is also necessary. But in general, as the following result shows, this is not true.

Theorem 1.4. Let $(S, \mathscr{S}, \mu)$ be a probability space, where $S$ is a Polish space with card $S=c, \mathscr{S}$ is the Borel $\sigma$-algebra and $\mu$ is a diffuse probability measure. Let $\mathscr{F}=\left\{F_{t} \mid t \in T\right\}$ be the class of subsets of $S$ that is established by Lemma 1.2 with $n=2$, and let $f: S \times T \rightarrow R$ be the function defined by

$$
f(s, t)=1_{F_{1}}(s) .
$$

Then the function $s \mapsto f(s)=f(s, \cdot)$ is an element of $\operatorname{LLN}\left(\mu, l_{2}(T)\right)$ but

$$
(s, v) \mapsto \eta(s, v)=\sum_{t \in T} f(s, t) f(v, t)
$$

is not $\mu \times \mu$ measurable. 
Proof. For every $s \in S$ we have that $f(s) \in l_{2}(T)$ because

$$
\|f(s)\|_{2}^{2}=\sum_{t \in T}|f(s, t)|^{2}=\sum_{t \in T}\left|1_{F_{t}}(s)\right|^{2}=\sum_{t \in T} 1_{F_{t}}(s)=1
$$

and this shows that $s \mapsto f(s)$ is a map from $S$ to $l_{2}(T)$.

We shall prove that

$$
\left\|\frac{1}{n} \sum_{i=1}^{n} f\left(s_{i}\right)\right\|_{2} \rightarrow 0 \quad \mu^{\infty}-\text { a.s. } \quad\left(s_{i}\right) \in S^{\infty}
$$

that is $f \in \operatorname{LLN}\left(\mu, l_{2}(T)\right)$ with mean 0 . We have

$$
\begin{aligned}
\left\|\frac{1}{n} \sum_{i=1}^{n} f\left(s_{i}\right)\right\|_{2}^{2} & =\frac{1}{n^{2}} \sum_{i=1}^{n} \sum_{j=1}^{n} \sum_{t \in T} f\left(s_{i}, t\right) f\left(s_{j}, t\right) \\
& =\frac{1}{n^{2}} \sum_{i=1}^{n} \sum_{j=1}^{n} \sum_{t \in T} 1_{F_{i}}\left(s_{i}\right) 1_{F_{i}}\left(s_{j}\right) \\
& =\frac{1}{n^{2}} \sum_{i=1}^{n} \sum_{j=1}^{n} 1_{F}\left(s_{i}, s_{j}\right)
\end{aligned}
$$

where

$$
F=\bigcup_{t \in T} F_{t} \times F_{t}
$$

If we define

$$
L=\left\{\left(s_{k}\right) \in S^{\infty} \mid s_{i} \neq s_{j}, \forall i, j, i \neq j\right\}
$$

then $\mu^{\infty}(L)=1$ because $\mu$ is diffuse. Since card $F_{t} \leqq 3$, we have for all $\left(s_{i}\right) \in L$

$$
\begin{aligned}
\left\|\frac{1}{n} \sum_{i=1}^{n} f\left(s_{i}\right)\right\|_{2}^{2} & =\frac{1}{n^{2}} \sum_{i=1}^{n} \sum_{j=1}^{n} 1_{F}\left(s_{i}, s_{j}\right) \\
& =\frac{1}{n^{2}} \sum_{i=1}^{n} \sum_{t \in T} 1_{F_{i}}\left(s_{i}\right) \sum_{j=1}^{n} 1_{F_{i}}\left(s_{j}\right) \\
& \leqq \frac{1}{n^{2}} \sum_{i=1}^{n} 3 \sum_{t \in T} 1_{F_{i}}\left(s_{i}\right)=\frac{3}{n}
\end{aligned}
$$


which shows that $f \in \operatorname{LLN}\left(\mu, l_{2}(T)\right)$. It remains to prove that the scalar product

$$
\eta\left(s_{1}, s_{2}\right)=\left(f\left(s_{1}\right), f\left(s_{2}\right)\right)=\sum_{t \in T} 1_{F_{1}}\left(s_{1}\right) 1_{F_{1}}\left(s_{2}\right)=1_{F}\left(s_{1}, s_{2}\right)
$$

is not a $\mu \times \mu$ measurable function. To this end, notice that if $s \in F_{u}$, for the section $F(s)$ we have

$$
\begin{aligned}
F(s)=\{v \in S \mid(v, s) \in F\} & =\left\{v \in S \mid(v, s) \in \underset{t \in T}{\bigcup} F_{t} \times F_{t}\right\} \\
& =\left\{v \in S \mid \exists t \in T:(v, s) \in F_{t} \times F_{t}\right\}=F_{u},
\end{aligned}
$$

and so $\mu(F(s))=0$ for all $s \in S$. If $A \subseteq F$ is a $\mu \times \mu$ measurable set, then $\mu(A(s))=0$ for every section $A(s), s \in S$, which implies that

$$
(\mu \times \mu)(A)=\int \mu(A(s)) \mu(d s)=0,
$$

and consequently $(\mu \times \mu)_{*}(F)=0$. If $B \in \mathscr{S}^{2}$ and $(\mu \times \mu)(B)>0$, then

$$
B \bigcap F=B \bigcap\left(\bigcup_{t \in T} F_{t} \times F_{t}\right)=\bigcup_{t \in T}\left(B \cap F_{t} \times F_{t}\right) \neq \varnothing
$$

which implies that $(\mu \times \mu)^{*}(F)=1$, and so $F$ and thus $\eta$ is not $\mu \times \mu$ measurable.

Remark. The family $\left\{F_{t} \mid t \in T\right\}$ has been chosen so that

$$
S=\bigcup_{t \in T} F
$$

which leads to

$$
\left\|\frac{1}{n} \sum_{i=1}^{n} f\left(s_{i}\right)\right\|_{2}^{2} \geqq \frac{1}{n}
$$

Therefore, for every $\left(s_{i}\right) \in L$ we have

$$
\sqrt{\frac{1}{n}} \leqq\left\|\frac{1}{n} \sum_{i=1}^{n} f\left(s_{i}\right)\right\|_{2} \leqq \sqrt{\frac{3}{n}}
$$


which implies that

$$
P\left\{1 \leqq\left\|\frac{1}{\sqrt{n}} \sum_{i=1}^{n} f\left(s_{i}\right)\right\|_{2} \leqq \sqrt{3}\right\}=1 .
$$

The Portmanteau theorem states that if

$$
\frac{1}{\sqrt{n}} \sum_{i=1}^{n} f\left(s_{i}\right)
$$

converge in law to some Gaussian Radon probability measure $\gamma$ on $l_{2}(T)$, then

$$
\underset{n \rightarrow \infty}{\lim \sup } P\left\{\left\|\frac{1}{\sqrt{n}} \sum_{i=1}^{n} f\left(s_{i}\right)\right\|_{2}<\alpha\right\} \geqq \gamma\left(B_{\alpha}\right)
$$

where $B_{\alpha}$ is an open ball with radius $\alpha$. But for $\alpha<1$

$$
P\left\{\left\|\frac{1}{\sqrt{n}} \sum_{i=1}^{n} f\left(\mathrm{~s}_{i}\right)\right\|_{2}<\alpha\right\}=0
$$

and thus $f$ does not satisfy CLT (Central Limit Theorem), but it does satisfy LLN.

The following theorem shows that there exist functions that have nonessentially separable valued range, but do satisfy LLN. Several other examples are known (see e.g. [5]).

Theorem 1.5. Let $(S, \mathscr{S}, \mu)$ be a probability space, where $S$ is a Polish space with card $S=c, \mathscr{S}$ is the Borel $\sigma$-algebra and $\mu$ is a diffuse probability measure. Then for any set $T$ with card $T \geqq c$ we have that $L^{1}(\mu, B)$ is the proper subspace of $\operatorname{LLN}(\mu, B)$, where $B$ can be any of the following spaces: $l_{p}(T)$ for $p \in(1, \infty]$ or $c_{0}(T)$.

Proof. It is no loss of generality to assume that card $T=c$. Let $F=\left\{F_{t} \mid t \in T\right\}$ be the class of subsets of $S$ that has been established by Lemma 1.2 with $n=1$, and let $f: S \times T \rightarrow R$ be the function defined by

$$
f(s, t)=1_{F_{1}}(s) .
$$

For every $s \in S, f(s)=f(s, \cdot)$ belongs to $l_{p}(T)$ for all $p \geqq 1$ and also $c_{0}(T)$. 
If we put

$$
L=\left\{\left(s_{k}\right) \in S^{\infty} \mid s_{i} \neq s_{j}, \forall i, j, i \neq j\right\},
$$

then $\mu^{\infty}(L)=1$ since $\mu$ is diffuse. For $\left(s_{i}\right) \in L$ we have

$$
\left\|\frac{1}{n} \sum_{i=1}^{n} f\left(s_{i}\right)\right\|_{p}^{p}=\sum_{t \in T}\left(\frac{1}{n} \sum_{i=1}^{n} 1_{F_{i}}\left(s_{i}\right)\right)^{p} \leqq n\left(\frac{2}{n}\right)^{p}=\frac{2^{p}}{n^{p-1}}
$$

for $p \geqq 1$ and

$$
\left\|\frac{1}{n} \sum_{i=1}^{n} f\left(s_{i}\right)\right\|_{\infty}=\sup _{t \in T}\left|\frac{1}{n} \sum_{i=1}^{n} 1_{F_{t}}\left(s_{i}\right)\right| \leqq \frac{2}{n},
$$

which shows that $f \in \operatorname{LLN}\left(\mu, l_{p}(T)\right)$ for all $1<p \leqq \infty$, and $f \in \operatorname{LNN}\left(\mu, c_{0}(T)\right)$. If $s \in F_{t}, s^{\prime} \in F_{t^{\prime}}$, and $1<p \leqq \infty$, then

$$
\left\|f(s)-f\left(s^{\prime}\right)\right\|_{p}=1 \text { if } t \neq t^{\prime} \text { and }\left\|f(s)-f\left(s^{\prime}\right)\right\|_{p}=0 \quad \text { if } t=t^{\prime}
$$

Let $N \in \mathscr{S}$. Then by (1.5.2), $f(S \backslash N)$ is separable if and only if $f(S \backslash N)$ is countable. By definition of $\left\{F_{t} \mid t \in T\right\} f$ maps at most two elements of $S \backslash N$ to the same element of $f(S \backslash N)$. Hence $f(S \backslash N)$ is countable if and only if $S \backslash N$ is countable, and since $\mu$ is diffuse, we have that if $f(S \backslash N)$ is separable, then $\mu(S \backslash N)=0$. Consequently $f$ does not have essentially separable valued range and therefore $f$ is not Bochner $\mu$-measurable.

Remark. For $p=1$ the function defined by (1.5.1) does not belong to $\operatorname{LLN}\left(\mu, l_{1}(T)\right)$. In [5, Theorem 2.4], its is shown that if $f \in \operatorname{LLN}$, then $f$ is Gelfand integrable. But if $f \in L_{G}^{1}\left(\mu, l_{1}(T)\right)$, then

$$
\int_{S} f(s) \mu(d s)=0
$$

since $f(\cdot, t)=0 \quad \mu$-a.s. for all $t \in T$. However, if $a_{t} \equiv 1$, then $a=\left(a_{t}\right) \in l_{\infty}(T)$ $=\left(l_{1}(T)\right)^{*}$ and

$$
\int_{S} a(f(s)) \mu(d s)=\int_{S} \sum_{t \in T} 1_{F_{t}}(s) \mu(d s)=1
$$


but

$$
a\left(\int_{S} f d \mu\right)=0 \neq 1
$$

so $f \notin L_{G}^{1}\left(\mu, l_{1}(T)\right)$ and consequently $f \notin \operatorname{LLN}\left(\mu, l_{1}(T)\right)$. With a bit more care one can even show that $f$ is not weakly measurable when we consider $f$ as an $l_{1}(T)$-valued random element.

\section{LLN for $\xi$-additive probability measures.}

In this chapter we shall consider $\xi$-additive measures as defined in [4]. In [5, Corollary 4.4$]$, it is proved that

$$
\operatorname{LLN}(\mu, C(T))=L_{*}^{1}(\mu, C(T)) \cap L_{w}^{1}(\mu, C(T))
$$

if $T$ is compact, first countable and separable. We shall now see that if $\mu$ is $\xi$-additive for some cardinal $\xi>\aleph_{0}$, then this result holds provided that the density and the character of $T$ are less than $\xi$. Recall that

$$
\text { dens } T=\min \{\operatorname{card} D \mid D \text { dense in } T\}
$$

$$
\operatorname{character}(T, t)=\min \{\operatorname{card} \mathcal{N} \mid \mathcal{N} \text { is neigbourhood base at } t\}
$$

whenever $T$ is a topological space and $t \in T$.

THeORem 2.1. Let $(S, \mathscr{S}, \mu)$ be a probability space, $\xi$ a cardinal with $\xi>\aleph_{0}$, and $\mu$ a $\xi$-additive measure. If $T$ is a compact topological space with dens $T<\xi$, character $(T, t)<\xi$ for all $t \in T$, then

$$
\operatorname{LLN}(\mu, C(T))=L_{*}^{1}(\mu, C(T)) \cap L_{w}^{1}(\mu, C(T)) .
$$

Proof. In [5, Theorem 2.4], it is proved that

$$
\operatorname{LLN}(\mu, B) \subseteq L_{w}^{1}(\mu, B) \cap L_{*}^{1}(\mu, B)
$$

for every Banach space $B$. We shall prove the reverse inclusion. Let $f \in L_{w}^{1}(\mu, C(T)) \cap L_{*}^{1}(\mu, C(T))$. Then

$$
\int_{S}^{*}\|f(s)\| \mu(d s)=\int_{S}^{*} \sup _{t \in T}|f(s, t)| \mu(d s)<\infty
$$


which implies that $f$ is a first order stochastic process. If we can prove that $f$ is totally bounded in $\mu$-mean, then by Theorem 3.3 in [5], we have finished. Therefore it remains to prove that

$$
\forall \varepsilon>0 \quad \exists \mathscr{A} \in \Gamma(T): \quad \int_{S}^{*} W_{A}(f(s)) \mu(d s)<\varepsilon, \quad \forall A \in \mathscr{A},
$$

where $\Gamma(T)=\{\mathscr{A} \mid \mathscr{A}$ is finite cover of $T\}$ and

$$
W_{A}(f)=\sup _{t, t^{\prime} \in A}\left|f(t)-f\left(t^{\prime}\right)\right|, \quad A \subseteq T .
$$

Since dens $T<\xi$, there exists $D \subseteq T$ such that card $D<\xi$ and $\bar{D}=T$. The functions $f(s): T \rightarrow R$ are continuous functions on $T$ for every $s \in S$, and therefore every open $U \subseteq T$ we have :

$$
\begin{gathered}
W_{U}(f(s))=\sup _{t, t^{\prime} \in U}\left|f(s, t)-f\left(s, t^{\prime}\right)\right|=\sup _{t, t^{\prime} \in U \cap D}\left|f(s, t)-f\left(s, t^{\prime}\right)\right| \\
=W_{U \cap D}(f(s)), \quad \forall s \in S .
\end{gathered}
$$

The family $\left\{s \mapsto\left|f(s, t)-f\left(s, t^{\prime}\right)\right| \mid t, t^{\prime} \in T\right\}$ is a family of $\mu$-measurable functions, so by $[4$, Corollary 3.2$]$, the functions

$$
S \mapsto U_{U \cap D}(f(s)), \quad U \subseteq T, \quad U \text { open, }
$$

are $\mu$-measurable since $\operatorname{card}(U \cap D)<\xi$. The inequality

$$
\left.W_{A}(f(s)) \leqq 2 \| f(s)\right) \|, \quad \forall s \in S \forall A \subseteq T,
$$

and (2.1.2) implies

$$
\int_{S}^{*} W_{A}(f(s)) \mu(d s)<\infty, \quad \forall A \cong T .
$$

\section{Hence}

$$
\int_{S} W_{U}(f(s)) \mu(d s)<\infty, \quad \forall U \subseteq T, U \text { open. }
$$


For fixed $t \in T$, we have that character $(T, t)<\xi$, and so there exists a downwards filtering family $\mathcal{N}$ of open neighbourhoods at $t$ such that card $\mathcal{N}<\xi$. Since $f(s, \cdot)$ is continuous at $t$, we have

$$
\inf _{N \in \mathcal{N}} W_{N}(f(s))=\inf _{N \in \mathcal{N}} \sup _{t, t^{\prime} \in N}\left|f(s, t)-f\left(s, t^{\prime}\right)\right|=0, \quad \forall s \in S,
$$

and by $[4$, Corollary 3.2$]$, we have

$$
\cdot \inf _{N \in \mathcal{N}} \int_{S} W_{N}(f(s)) \mu(d s)=\int_{N} \inf _{N \in \mathcal{N}} W_{N}(f(s)) \mu(d s)=0,
$$

which implies that for every $\varepsilon>0$ there exist $N(t) \in \mathcal{N}$ such that

$$
\int_{N} W_{N(t)}(f(s)) \mu(d s)<\varepsilon .
$$

Since the family $\mathscr{M}=\{N(t) \mid t \in T\}$ is an open cover of $T$, the compactness of $T$ ensures that there exists a finite subset $\mathscr{A}=\left\{N\left(t_{i}\right) \mid i=1, \ldots, n\right\}$ of $\mathscr{M}$ such that $\mathscr{A}$ is a cover of $T$ and such that

$$
\int_{S} W_{N\left(t_{i}\right)}(f(s)) \mu(d s)<\varepsilon \quad \forall i, i=1, \ldots, n .
$$

Recall that a function $f:(S, \mathscr{S}, \mu) \rightarrow B$ is Bochner $\mu$-measurable if $f$ is weakly $\mu$-measurable and $f$ has $\mu$-essentially separable range, that is, if there exists a $\mu$-null set $N$ such that $f(S \backslash N)$ is separable. The $\xi$-additive measures give us an opportunity to extend the definition of Bochner measurability in the following way: A weakly $\mu$-measurable function $f:(S, \mathscr{S}, \mu) \rightarrow B$ is $\xi$-Bochner $\mu$-measurable if there exists a $\mu$-null set $N$ such that dens $f(S \backslash N)<\xi$. But it turns out, as we show below, that we do not get more than the usual Bochner measurability.

Theorem 2.2. Let $(S, \mathscr{S}, \mu)$ be a probability space, where $\mu$ is a $\xi$-additive measure with $\xi>\aleph_{0}$, and let $f:(S, \mathscr{S}, \mu) \rightarrow B$ be a weakly measurable function. If there exists a $\mu$-null set $N$ such that dens $f(S \backslash N)<\xi$, then $f$ is Bochner $\mu$-measurable.

Proof. Let $D=\left\{x_{\alpha} \mid \alpha<\Gamma\right\}$ be a subset of $B$ such that $f(S \backslash N) \subseteq \bar{D}$ and 
$\Gamma<\xi$. Since $\xi>\boldsymbol{N}_{0}$, it is no loss of generality to assume that $D$ is a vector space over the rationals, and so that $\bar{D}$ is a closed linear subspace of $B$. By the Hahn-Banach theorem there exist functionals $x_{\alpha}^{*} \in B^{*}, \alpha<\Gamma$, such that

$$
\left\|x_{\alpha}\right\|=x_{\alpha}^{*}\left(x_{\alpha}\right) \text { and }\left\|x_{\alpha}^{*}\right\|=1, \quad \forall \alpha<\Gamma .
$$

We claim that

$$
\|x\|=\sup _{\alpha<\Gamma} x_{\alpha}^{*}(x), \quad \forall x \in \bar{D} .
$$

The inequality $\geqq$ is obvious. So let $x \in \bar{D}$ and let $\varepsilon>0$ be given. Then there exists $\alpha<\Gamma$ with $\left\|x-x_{\alpha}\right\|<\varepsilon$, and so

$$
\|x\| \leqq \varepsilon+\left\|x_{\alpha}\right\|=\varepsilon+x_{\alpha}^{*}\left(x_{\alpha}\right)=\varepsilon+x_{\alpha}^{*}(x)+x_{\alpha}^{*}\left(x_{\alpha}-x\right) \leqq 2 \varepsilon+x_{\alpha}^{*}(x) .
$$

Thus the converse inequality in (2.2.2) holds. From (2.2.2) we have that

$$
\left\|f(s)-x_{\beta}\right\|=\sup _{\alpha<\Gamma} x_{\alpha}^{*}\left(f(s)-x_{\beta}\right), \quad \forall \beta<\Gamma, \quad \forall s \in f(S \backslash N)
$$

since $\bar{D}$ is a vector space containing $f(s)$ and $x_{\beta}$. By Corollary 3.2 in [4], the function

$$
s \mapsto\left\|f(s)-x_{\beta}\right\|
$$

is $\mu$-measurable for all $\beta<\Gamma$ and since $f(S \backslash N) \cong \bar{D}$, we have

$$
S \backslash N \cong \bigcup_{\beta<\Gamma}\left\{s \mid\left\|f(s)-x_{\beta}\right\|<\varepsilon\right\}, \quad \forall \varepsilon>0 .
$$

By [4, Theorem 3.1], there exist a countable set $\Lambda \subseteq\{\alpha \mid \alpha<\Gamma\}$ and a null set $N_{0} \supseteqq N$ such that

$$
S \backslash N_{0} \subseteq \bigcup_{\beta \in A}\left\{s \mid\left\|f(s)-x_{\beta}\right\|<2^{-n}\right\}, \quad \forall n \geqq 1
$$

Hence $f\left(S \backslash N_{0}\right) \subseteq \overline{\left\{x_{\beta} \mid \beta \in \Lambda\right\}}$, and so $f$ is essentially separably valued. Thus $f$ is Bochner $\mu$-measurable.

Proposition 2.3. Let $(S, \mathscr{S}, \mu)$ be a probability space, where $\mu$ is a $\xi$-additive measure with $\xi>\aleph_{0}$; let $B$ be a Banach space, and let $f:(S, \mathscr{S}, \mu) \rightarrow B$ be a 
function such that dens $f(S \backslash N)>\xi$ for some $\mu$-null set $N$. Then $f \in \operatorname{LLN}(\mu, B)$ if and only if $f \in L_{*}^{1}(\mu, B) \cap L_{w}^{1}(\mu, B)$.

Proof. If $f \in \operatorname{LLN}(\mu, B)$, then by [5, Theorem 2.4], we have that $f \in L_{*}^{1}(\mu, B) \cap L_{w}^{1}(\mu, B)$. If $f \in L_{w}^{1}(\mu, B)$ and dens $f(S \backslash N)>\xi$ for some $\mu$-null set $N$, then by Theorem 2.2, $f$ is Bochner $\mu$-measurable and $f \in L_{*}^{1}(\mu, B)$ ensures that $f$ is Bochner $\mu$-integrable; but then $f \in \operatorname{LLN}(\mu, B)$ by the wellknown result due to $A$. Beck $([1$, p. 26$])$.

Corollary 2.4. Let $(S, \mathscr{S}, \mu)$ be a probability space, where $\mu$ is a $\xi$-additive measure with $\xi>\aleph_{0}$, and let $T$ be a set such that card $T<\xi$. Then

$$
\operatorname{LLN}\left(\mu, l_{p}(T)\right)=L_{w}^{1}\left(\mu, l_{p}(T)\right) \cap L_{*}^{1}\left(\mu, l_{p}(T)\right)
$$

for $1 \leqq p<\infty$.

Proof. This is an immediate consequence of Proposition 2.3 because

$$
\operatorname{dens} l_{p}(T) \leqq \operatorname{card} T \cdot \aleph_{0}<\xi \aleph_{0}=\xi,
$$

and therefore $\operatorname{dens} f(S)<\xi$.

\section{Functions which do not satisfy LLN.}

In [3] E. Giné and J. Zinn give a necessary and sufficient condition for $f \in \operatorname{LLN}\left(\mu, l_{\infty}(T)\right)$ provided that $f$ satisfies some measurability condition (condition $\operatorname{NSM}(P)$ in [3]). We shall describe this necessary and sufficient condition. Let $(S, \mathscr{S}, \mu)$ be a probability space, $T$ some set and $f: S \times T \rightarrow \mathrm{R}$ a stochastic process. Consider the random metrics defined by

$$
\begin{gathered}
d_{n, p}\left(t, t^{\prime}\right)=\left(\frac{1}{n} \sum_{i=1}^{n}\left|f\left(s_{i}, t\right)-f\left(s_{i}, t^{\prime}\right)\right|^{p}\right)^{1 / p \vee 1} \text { for } 0<p<\infty, \\
d_{n, \infty}\left(t, t^{\prime}\right)=\max _{1 \leqq i \leqq n}\left|f\left(s_{i}, t\right)-f\left(s_{i}, t^{\prime}\right)\right|
\end{gathered}
$$

for all $t, t^{\prime} \in T$, their covering numbers $N_{n p}(r, f)$ (i.e. the smallest integer $N$ such that $T$ may be covered by $N, d_{n, p}$-balls of radius $r$ ), and their metric entropies $H_{n, p}(r, f)$ defined by

$$
H_{n, p}(r, f)=\ln N_{n, p}(r, f) \text {. }
$$

Then under a certain measurability condition it is shown in [3] that 
$f \in \operatorname{LLN}\left(\mu, l_{\infty}(T)\right)$ if and only if

$$
s \mapsto F(s)=\sup _{t \in T}|f(s, t)| \text { belongs to } L^{1}(\mu)
$$

and

$$
\lim _{n \rightarrow \infty} E^{*} \frac{H_{n, p}\left(r, f 1_{[F \leqq M]}\right)}{n}=0, \quad \forall r>0, \forall M>0, \forall p \in(0, \infty]
$$

We shall show that there exists a function $f$ satisfying (3.4) and (3.5), but $f \notin \operatorname{LLN}\left(\mu, l_{\infty}(T)\right)$, hence the measurability condition in [3] is to be necessary for the entropy condition to imply $\operatorname{LLN}\left(\mu, l_{\infty}(T)\right)$. Moreover, this function is Gelfand integrable which shows that in general $\operatorname{LLN}(\mu, B)$ is a proper subspace of $L_{G}^{1}(\mu, B) \cap L_{*}^{1}(\mu, B)$.

Theorem 3.1. Let $(S, \mathscr{S}, \mu)$ be a probability space, where $S$ is a Polish space with card $S=c, \mathscr{S}$ is the Borel $\sigma$-algebra and $\mu$ is a diffuse probability measure. Let $\mathscr{F}=\left\{F_{t} \mid t \in T\right\}$ be the class of subsets of $S$ that is established by Lemma 1.1 , and let $f: S \times T \rightarrow R$ be the function defined by

$$
f(s, t)=1_{F_{t}}(s) .
$$

Then

$$
f \in L_{*}^{1}(\mu, B) \cap L_{G}^{1}(\mu, B),
$$

$$
f \notin \operatorname{LLN}(\mu, B) \text {, }
$$

$f$ satisfies the entropy condition (3.4) and (3.5),

$$
N_{n, p}(r, f) \leqq n+1, \quad \forall n \geqq 1, \forall r \geqq 0, \forall 0<p \leqq \infty,
$$

where $B$ is any of the following Banach spaces

$$
l_{p}(T) \text { for } 1<p \leqq \infty \text { or } c_{0}(T)
$$

Proof. Since

$$
S=\bigcup_{t \in T} F_{t}
$$

we have

$$
\sum_{t \in T}|f(s, t)|^{p}=\sum_{t \in T} 1_{F_{t}}(s)=1, \quad \forall s \in S, \forall 1 \leqq p<\infty
$$


which implies that functions

$$
f(s): t \mapsto 1_{F_{t}}(s)
$$

belong to $l_{p}(T),\|f(s)\|_{p}=1$ for all $s \in S$ and all $p, 1 \leqq p<\infty$, and that

$$
\int_{s}^{*}\|f(s)\|_{p} \mu(d s)=\int_{s}\|f(s)\|_{p} \mu(d s)=1, \quad \forall 1 \leqq p<\infty .
$$

Hence $f \in L_{*}^{1}\left(\mu, l_{p}(T)\right)$ for all $1 \leqq p<\infty$. For every $s \in S$ there exists exactly one $t$ such that $1_{F_{t}}(s)=1$, showing that $f(s) \in c_{0}(T)$ for every $s \in S$ and therefore that $f \in L_{*}^{1}\left(\mu, c_{0}(T)\right) \subseteq L_{*}^{1}\left(\mu, l_{\infty}(T)\right)$.

Now we shall show that functions $s \mapsto f(s)$ belong to $L_{G}^{1}\left(\mu, l_{p}(T)\right)$ for $1<p \leqq \infty$ and to $L_{G}^{1}\left(\mu, c_{0}(T)\right)$. Let $1<p<\infty$; let $q$ be such that

$$
\frac{1}{p}+\frac{1}{q}=1
$$

and let $x=\left(x_{t}\right) \in l_{p}^{*}(T)=l_{q}(T)$. Then

$$
\sum_{t \in T}\left|x_{t}\right|^{q}<\infty
$$

implying that the set $T_{0}=\left\{t \mid x_{t} \neq 0\right\}$ is countable. If

$$
M=\left\{s \in S \mid x(f(s))=\sum_{t \in T} x_{t} 1_{F_{t}}(s) \neq 0\right\}
$$

then

$$
M \subseteq \bigcup_{t \in T_{0}} F_{t}
$$

Since $F_{t}$ and $T_{0}$ are countable, then so is $M$. The measure $\mu$ is diffuse, and therefore $\mu(M)=0$, which implies that $x(f(\cdot))$ is $\mu$-measurable and

$$
\int_{S} x(f(s)) \mu(d s)=0
$$


for every $x \in l_{p}^{*}(T)$ and every $1<p<\infty$. Thus

$$
\int_{S} f(s) \mu(d s)=0 \quad \text { and } \quad f \in L_{G}^{1}\left(\mu, l_{p}(T)\right), \quad \forall p, 1<p<\infty .
$$

The same proof goes for $c_{0}(T)$ because $c_{0}(T)^{*}=l_{1}(T)$. The space $c_{0}(T)$ can be embedded naturally into $l^{\infty}(T)$. For every $x \in l^{\infty}(T)^{*}$ we define

$$
x^{\prime}=\left.x\right|_{c_{0}(T)} .
$$

Then $x^{\prime} \in l_{1}(T)$ and

$$
x^{\prime}(f(s))=x(f(s)), \quad \forall s \in S .
$$

Now working with $x^{\prime}$ we conclude that

$$
\int_{S} x^{\prime}(f(s)) \mu(d s)=0
$$

and from (3.1.8) and (3.1.7) we conclude that

$$
\int_{S} f(s) \mu(d s)=0 \text { and } f \in L_{G}^{1}\left(\mu, l_{\infty}(T)\right) \cap L_{G}^{1}\left(\mu, c_{0}(T)\right) .
$$

So we have proved (3.1.2). To prove (3.1.3), let

$$
L_{p}^{\infty}=\left\{\left(s_{i}\right) \in S^{\infty} \mid\left\|\frac{1}{n} \sum_{i=1}^{n} f\left(s_{i}\right)\right\|_{p}=1, \forall n \in N\right\}, \quad 1<p \leqq \infty .
$$

Then by (1.1.2) we have for all $1<p \leqq \infty$

$$
\begin{aligned}
L_{p} & =\left\{\left(s_{i}\right) \in S^{\infty} \mid \exists t \in T: \frac{1}{n} \sum_{i=1}^{n} 1_{F_{i}}\left(s_{i}\right)=1, \forall n \in N\right\} \\
& =\left\{\left(s_{i}\right) \in S^{\infty} \mid \exists t \in T:\left\{s_{i} \mid i \in N\right\} \cong F_{t}\right\},
\end{aligned}
$$

and it now easily follows that

$$
L_{p}=\bigcup_{t \in T} F_{t}^{\infty}, \quad 1<p \leqq \infty
$$


By (1.1.3) we have $B \cap L_{p} \neq \varnothing$ for every $B \in \mathscr{S}^{\infty}$ with $\mu^{\infty}(B)>0$, which implies

$$
\left(\mu^{\infty}\right)^{*}\left(L_{p}\right)=1
$$

and so (3.1.3) follows.

To prove (3.1.4), it is enough to verify (3.5) for $M=1$. Let $r>0$, $n \geqq 1,0<p \leqq \infty$ and $\left(s_{j}\right) \in S^{\infty}$ be given. Choose $t_{j} \in T, j=1, \ldots, n$, such that $s_{j} \in F_{t_{j}}($ see $(1.1 .4))$, and let

$$
U_{n}=T \backslash\left\{t_{1}, \ldots, t_{n}\right\}
$$

If $u, v \in U_{n}$, then

$$
1_{F_{u}}\left(s_{j}\right)=1_{F_{v}}\left(s_{j}\right)=0, \quad \forall j=1, \ldots, n .
$$

So we have that $U_{n}$ is a $d_{n, p}$-ball of radius 0 . Clearly $\left\{t_{j}\right\}$ for $j=1, \ldots, n$ are $d_{n, p}$-balls of radius 0 . Hence

$$
N_{n, p}(r, f) \leqq N_{n, p}(0, f) \leqq n+1, \quad \forall n \geqq 1, \forall r \geqq 0, \forall 0<p \leqq \infty
$$

since $T=U_{n} \bigcup \bigcup_{i=1}^{n}\left\{t_{i}\right\}$. In particular we have

$$
\lim _{n \rightarrow \infty} E^{*} \frac{H_{n, p}(r, f)}{n} \leqq \lim _{n \rightarrow \infty} \frac{\log (n+1)}{n}=0
$$

\section{REFERENCES}

1. A. Beck, On the strong law of large numbers, in Ergodic Theory, (Proc. Sympos., New Orleans, 1961), pp. 21-53. Academic Press, New York, 1963.

2. Y.S. Chow and H. Teicher, Probability Theory. Independence, Interchangeability, Martingales. Springer-Verlag, Berlin - Heidelberg - New York, 1978.

3. E. Giné and J. Zinn, Some limit theorems for empirical processes, Ann. Probab. 12 (1984), 929-989.

4. J. Hoffmann-Jørgensen, How to make a divergent sequence convergent by Martin's axiom, Mat. Inst., Aarhus Univ., Preprint Series No. 21, 1977.78.

5. J. Hoffmann-Jørgensen, The law of large numbers for non-measurable and non-separable random elements, (Coll., Ecole Polytechnique, Paris, 1983), eds. M. R. Herman and B. Teissier (Astérisque 131), pp. 279-298. Société Mathématique de France, Paris, 1985.

6. M. Talagrand, The Glivenko-Cantelli problem, Preprint, Ohio State Univ., 1984.

MATEMATISK INSTITUT AARHUS UNIVERSITET NY MUNKEGADE 8000 AARHUS C DENMARK and

THE CITY COLLEGE OF THE CITY UNIVERSITY OF NEW YORK DEPARTMENT OF MATHEMATICS NEW YORK, N.Y. 10031 U.S.A. 